\title{
La investigación y el proceso de elaboración de Trabajos Finales de Graduación: una propuesta desde la maestría en Derecho del Trabajo y Seguridad Social
}

\section{Fabiola Cantero Acosta ${ }^{1}$ \& Carlos H. Ulate Ulloa ${ }^{2}$}

1. Directora de la Maestría en Derecho del Trabajo y Seguridad Social de la Universidad Estatal a Distancia (UNED). Master en esa disciplina y Doctoranda en Derecho de la UNED. Miembro de la Comisión de Derecho Laboral y del Comité de Excelencia Académica del Colegio de Abogados y Abogadas de Costa Rica. Miembro de la Red de Investigación para el Desarrollo del Conocimiento y Propuestas Tecnológicas Innovadoras aplicables a la Educación Superior a Distancia, de la Red de Cambio Climático de la UNED, así como del Comité Científico de la Revista Nacional de Administración. Profesora e investigadora universitaria.

2. Master en Ciencias de la Educación con Énfasis en Administración Educativa de la ULASALLE. Master en Educación en Valores de la Universidad de Barcelona, España. Profesor e investigador, actualmente Coordinador de Trabajos Finales de Graduación de la Escuela de Ciencias Sociales y Humanidades de la Universidad Estatal a Distancia (UNED).

Recibido: Enero 2015 • Aceptado: Febrero 2015

\section{RESUMEN}

El artículo plantea un repaso de la situación de la investigación en la maestría en Derecho del Trabajo y Seguridad Social de la Universidad Estatal a Distancia. Se presentan los antecedentes y las acciones remediales que desde la dirección de la maestría se han tomado para tratar de subsanar la situación final de los estudiantes egresados. Además, a manera de cierre, se exponen los primeros resultados que se han dado a partir de la implementación de tales acciones.

Palabras clave: investigación, Derecho del Trabajo, seguridad social, tesis, proyecto

\section{ABSTRACT}

The article presents an overview of the status of research in the master's degree in Labor Law and Social Security of the Universidad Estatal a Distancia (UNED), in Costa Rica. Specifically, the the author presents historical facts and procedures taken by the master's degree administration to try to resolve the final status of graduate students. Also, this paper explains the results obtained by the implementation of those administrative solutions.

Key words: research, Labour Law, Social Security, dissertation, academical project.

\section{Introducción}

\section{Antecedentes}

La maestría en Derecho del Trabajo y Seguridad Social (MDTSS) es un posgrado profesional de la Escuela de Ciencias Sociales y
Humanidades, adscrito al Sistema de Estudios de Posgrado. Fue creada en el año 2000 y es el único posgrado en esta disciplina en la región centroamericana, México y el Caribe. Su plan de estudios tiene una duración de cuatro cuatrimestres, con uno adicional, en el que se presenta un Trabajo Final de Graduación (TFG), cuya defensa 
pública aprobada otorga al postulante el grado de magister en la disciplina. El TFG, además, es el resultado final de un proceso de investigación disciplinar, en virtud del cual se entiende que la persona postulante es un especialista en el área.

Actualmente, se han abierto diez cohortes, la décimo primera recién finalizó en el 2014. Hasta la novena, que comprendió los años 2011-2012, habían pasado por este programa 203 personas ${ }^{1}$, 126 de las cuales han finalizado su plan de estudios y 85 se han graduado; con una media de integrantes por grupo de 22, personas. Debe añadirse que la MDTSS se abre cada vez que un grupo finaliza, y que en cada cuatrimestre se cursan cuatro materias.

Estos datos son producto de dos investigaciones (Umaña, 2011 y Umaña y Vargas, 2012). En el primer estudio, el objetivo principal fue determinar las causas que inciden en el rendimiento académico, la permanencia, abandono o conclusión de los estudios de las personas estudiantes matriculadas en la maestría en Derecho del Trabajo y Seguridad Social. En el segundo, se hizo una actualización de los resultados que ya se habían obtenido, pero introduciendo las variables propias de las acciones de mejora implementadas, para atender en un primer nivel las deficiencias y necesidades reveladas en el 2011. Así, dentro de una lógica de gestión de ese posgrado, se dio un diagnóstico cuyos resultados generaron acciones, que, con el segundo estudio fueron evaluadas.

\section{En específico: trabajos finales de graduación (TFG) y graduación}

Uno de los resultados más alarmantes de los estudios indicados fue el alto número de personas egresadas, con el TFG pendiente de elaborar para graduarse. Esto significa que muchas personas estudiantes terminaban su plan de estudios y no dan el último paso, la investigación que los acreditaba como especialistas disciplinares y que también se identifica con el aporte final que la Universidad hace a la sociedad, si se toma en cuenta que el éxito de una oferta académica se mide por el número de personas graduadas que tenga. Además, en el caso de un posgrado, ese impacto también está asociado a la investigación que aporte a su disciplina, la MDTSS no estaba siendo lo exitosa que podía y debía ser.

\section{Los datos}

¿Cuáles eran las razones para que el número de personas egresadas fuera tan alto, en relación con las potenciales graduaciones? ¿Quiénes eran esas personas? Para empezar, vale indicar que el $51,8 \%$ de las personas estudiantes es mujer, menor de 40 años, que vive en la Gran Área Metropolitana. Proviene de universidades privadas, en su mayoría, y trabaja en condición de propiedad o estabilidad. Los varones comparten las mismas características, exceptuando el rango etario, pues el $63 \%$ de los hombres que ha matriculado la MDTSS es mayor de 40 años.

Un dato revelador resultó ser que las mujeres dedican un promedio de treinta horas semanales a sus estudios en la maestría, en tanto que los varones, solamente once. De la misma manera, ellas tienden a permanecer más en el programa que ellos, pues del total matriculado, el porcentaje de abandono de las mujeres es del $38 \%$ y el de los varones del $45 \%$.

Los porcentajes de graduación tienden a ser más altos y en una menor cantidad de tiempo en el caso de las mujeres. Del total de las que han ingresado, un 44,9\% se ha graduado, mientras que en el caso de los hombres, ese porcentaje resulta de apenas el $37,6 \%$ del total de los matriculados. Aunado a esto, un $62,26 \%$ del total de mujeres que se han graduado invirtió menos de 25 meses entre ingresar a la Maestría y defender su TFG; en el caso de los hombres este porcentaje desciende a 37,5\%. (Umaña et al, 2012: 45)

Finalmente, de interés para este apartado, debe agregarse que los cursos de "Estrategias de Investigación Jurídico Laboral I" (EIJL1) y "Estrategias de Investigación Jurídico Laboral II" (EIJL2) fueron los cursos que menos agradaron a las personas estudiantes. Estas dos materias son las que, se supone, preparan la elaboración y defensa del TFG, para una posterior graduación. 


\section{Las acciones}

Ante los datos obtenidos, se desarrollaron las siguientes acciones:

- Se emprendió un plan para la recuperación de los egresados, que incluyó contactarlos a todos y girarles la invitación para que retomaran sus procesos de graduación dentro de un "período ventana" que se abrió para ellos, con un protocolo de investigación transicional diseñado al efecto. Además, se dio la revisión de cada uno de los casos y el diseño de soluciones académicas más personalizadas (que podía incluir cambio de tema de la investigación, si fuera necesario), cronogramación y fijación tentativa de fechas de defensa. Una vez consensuado lo anterior con cada egresado, se dio un fuerte acompañamiento, supervisión y rendición de cuentas de los resultados esperados, de conformidad con lo planificado. Con miras a que el rezago de egresados no se presente en el futuro, también se varió el contenido curricular de los cursos de investigación del plan de estudios, su orden en la oferta académica y el perfil del docente a cargo. Adicionalmente, se mejoró el protocolo de investigación, con base en las experiencias vividas por los rezagados y la proyección de graduación de las cohortes, a partir de la novena. (Cantero, 2013)

- El plan de estudios de la MDTSS tiene sus cursos articulados, pero no concatenados, en forma tal que cada materia es una unidad en sí misma. Esto permitió reacomodar varias de ellas, según una secuencia más fluida. Dentro de esos ajustes, se contempló la ubicación curricular de "Estrategias de Investigación Jurídico Laboral I y II". Originalmente, estaban situadas en el primer y cuarto cuatrimestre del plan, lo que derivaba en una interrupción del proceso de investigación y redacción del informe del TFG. Se procedió pues a ubicar el curso EIJL1 en el tercer cuatrimestre, de manera que los dos espacios formales de investigación tuvieran un continum en dos cuatrimestres seguidos.
- En virtud de que la normativa interna de la universidad permite la programación de actividades extraordinarias dentro del cuatrimestre, y dado que en el primer y segundo período, al desplazar el curso EIJL1, se creaba un espacio en que no había investigación como materia formal, se procedió a diseñar cuatro "Talleres de Pre-investigación", dos en cada uno de los cuatrimestres.

- En esos espacios, el docente a cargo de los cursos de EJL1 Y EJL2, en conjunto con la Dirección de la MDTSS, trabajan con las personas estudiantes su propuesta de investigación para el TFG. En este sentido, se les dota de las herramientas epistemológicas, teóricas y metodológicas básicas para el desarrollo y adecuado planteamiento de su tema de investigación, el problema a investigar, el objetivo general, los objetivos específicos, el estado de la cuestión, la justificación y una revisión primaria de referencias bibliográficas. El resultado final de este proceso es el documento de partida que la persona estudiante debe presentar en la primera clase de EJL1.

- Se asigna a la persona estudiante un acompañamiento formal de un director o directora de su TFG desde el momento en que tiene el anterior documento de partida avalado. Idealmente, durante el segundo cuatrimestre, nunca más allá de la fecha de inicio del tercero.

- Se determinó un protocolo de elaboración y defensa del TFG. Este incluye deberes de la persona estudiante, funciones de su Comité Asesor, estructura formal del informe, tipos de informe, entre otros aspectos operativos.

- Se estableció un período límite para poder defender el TFG, mismo que se fijó en el cuatrimestre inmediato siguiente a la finalización de la aprobación de todos los cursos del plan de estudios. Extraordinariamente, es prorrogable por una única vez.

- Se empezaron a fortalecer los espacios de divulgación de las investigaciones de las personas estudiantes que originaban sus TFG. 
Lo anterior surge a partir del reconocimiento de tres momentos importantes que se desarrollan en todo proceso de investigación adscrito a una carrera, y que la maestría ha tenido presente, a saber:

\section{Un momento ontológico}

(El objeto de la investigación)

Desde la constitución de la carrera, la misma se ha dado a la tarea de identificar claramente cuáles son sus líneas de trabajo, las cuales apuntan grosso modo al Derecho del Trabajo y a la Seguridad Social. A partir de estas se espera que el estudiante reflexione e identifique su área de interés, para que pueda iniciar el proceso de problematización de la realidad. La gran temática desarrollada por los estudiantes en sus propuestas de investigación en las últimas cohortes (2012 - 2015) demuestra la riqueza y la amplitud del objeto de estudio que trata la maestría. Actualmente se sigue en la exploración y profundización de esa rica veta para que de ella surjan objetos, temáticas a investigar que impacten, que sean actuales y que respondan al contexto que en Derecho del Trabajo y Seguridad Social requiera ser tratado, fortalecido y expuesto ante la comunidad nacional.

\section{Un momento epistemológico: ¿se descubre o se construye el conocimiento?}

La investigación de la sociedad, la cultura y el desarrollo desde un marco de referencia como lo son las Ciencias Sociales y Humanidades apunta a que en la maestría en Derecho del Trabajo y Seguridad Social reconozca máximas como las siguientes:

- No todo conocimiento científico se da por la información que se reciba empíricamente. Esta por lo general se presenta bajo aspectos cuantificables, los cuales son sesgados por una sociedad que ha apostado por una perspectiva racional exacerbada. Mucha de la investigación actual plantea que aquello que no sea cuantificable no puede entrar en los cánones de la razón; por tanto, no puede considerarse como conocimiento científico.

A partir de lo anterior, en estricto sentido, debe tenerse presente que las temáticas que pueden surgir del pensum de la maestría pueden perfectamente ocuparse de lo subjetivo en el ser humano. De tal forma que se deja por fuera la evidencia empírica cuantificable fuera de su alcance.

No obstante, desde el punto de vista epistemológico, la investigación científica en la maestría hace que el investigador apele a la elección de un método que le permita dar el tratamiento adecuado al objeto de estudio de su interés. Al respecto, Abello Llanos plantea que:

(...) las preguntas más frecuentes sobre la metodología son las siguientes: ¿cuál es el tipo y diseño que se proponen para la investigación y cuál es su fundamentación? ¿Cómo y porqué se escogió una determinada población o muestra? ¿Cuáles son las técnicas e instrumentos de investigación? ¿Cuál es la validez y confiabilidad de las técnicas? ... estas interrogantes deben ser resueltas con criterios de objetividad, rigor y precisión. (2009: 223)

Estas preguntas llevan al investigador a posicionarse frente al objeto de estudio y tomar apuesta a favor de una posición epistemológica determinada. He ahí el dilema: cuantificar o cualificar. Surge entonces, desde la perspectiva paradigmática, positivista o naturalista, los distintos diseños de investigación que puede asumir el investigador.

Ahora bien, el asumir una perspectiva paradigmática positivista ha dado como resultado la adopción de diseños sumamente estructurados, enmarcados en un procedimiento de índole deductivo, donde los experimentos, cuasi-experimentos y la investigación no experimental marcan la pauta. Por otro lado, una perspectiva paradigmática naturalista plantea la adopción de diseños flexibles, sumamente participativos, de corte inductivo. Tales como lo etnográfico, los estudios hermenéuticos o de análisis de contenido, el estudio de casos, la investigación acción, los estudios fenomenológicos, entre otros. 
El estudio de los fenómenos en las Ciencias Sociales y las Humanidades puede tomar en cuenta cualquiera de las perspectivas clásicas de producción de conocimiento. Se ha de tener presente que ninguno de los dos es superior respecto al otro y que de ambos el conocimiento nuevo que se obtenga será conocimiento científico.

De hecho, un vistazo a investigaciones realizadas en Ciencias Sociales en los últimos años permiten observar la utilización de propuestas donde se utilizan distintos métodos (multi-métodos) desde posicionamientos epistemológicos que antes se consideraban antagónicos y hoy día se ven como complementarios. La utilización de diseños de corte cuantitativos, ya sea cuasi-experimentales o no experimentales, con acercamientos puntualmente cualitativos como las etnografías, la teoría fundamentada, entre otros da como resultado un acercamiento más integral, más holista a los objetos de estudio, que por lo general son ricos en la posibilidad de aprehenderlos. Lógicamente, los resultados que se logran con este tipo de abordajes (multi-métodos) permiten la obtención de explicaciones a partir del establecimiento de relaciones causa-efecto y además la interpretación que lleva a la comprensión de las subjetividades del grupo estudiado.

\section{Un momento axiológico (La ética en la investigación)}

La premisa de partida de esta sección apela a que la investigación en la maestría en Derecho del Trabajo y Seguridad Social adscrita a la Escuela de Ciencias Sociales y Humanidades trata directamente con el ser humano. Por esto, la siguiente pregunta que surge apunta respecto a ¿para qué se realiza la investigación en la maestría? Se obtiene una respuesta que siempre será la necesidad de la producción de nuevo conocimiento. Ahora bien, ¿qué hace el investigador para que este nuevo conocimiento sea verdadero, apegado a la realidad que estudió y que llegue a ser conocido por la comunidad nacional?

Para dar respuesta a esta última pregunta se ha de reconocer que hay dos aspectos importantes que entran en juego en este momento. Al respecto, como plantea Ramírez Caro (2011) que la investigación debe hacerse de una manera correcta, no solo desde el punto de vista técnico, sino también desde el punto de vista ético. El conocimiento obtenido de todo el proceso de investigación realizada, sea en el área de las Ciencias Exactas y Naturales o en el área de las Ciencias Sociales y Humanidades, debe ser utilizado de manera responsable. Lo anterior señala a aspectos que deben ser considerados por el investigador desde una perspectiva ética, como los siguientes:

- Desde el acercamiento epistemológico el investigador utilizará aquellos métodos o diseños de investigación que sean apropiados para dar un tratamiento lo más adecuado al objeto de estudio.

- Existe respeto, por parte del investigador hacia los sujetos que participan en el estudio. Los sujetos no deben ser vistos como objetos a partir de los cuales se obtiene información. Sobre todo debe ser respetada su decisión de no participación en el estudio a realizarse.

- El plagio es una práctica que debe ser erradicada desde los procesos iniciales de formación estudiantil y es inexcusable cuando aparece en documentos académicos.

- La manipulación de los datos se presenta cuando a toda costa el investigador "desea cuadrar" los resultados a sus premisas personales iniciales. Los datos que han sido manipulados llevan a conclusiones y recomendaciones erradas que pueden afectar a la sociedad.

Según lo anterior, la ética en la investigación de la sociedad, la cultura y el desarrollo debe estar presente en todo momento. No es aceptable que por razones políticas o financieras el investigador se desligue de la ética. En todo momento la verdad debe salir a relucir. Sean cual fueren los resultados obtenidos el investigador debe ser lo más objetivo posible. Solo así, podrá comunicar resultados ciertos a la hora de compartir sus hallazgos con el grupo humano que investigó. 


\section{A manera de cierre}

A partir de los aspectos arriba señalados y tomando en cuenta las propuestas de investigación que han surgido en el seno de la ECSH, puede argumentarse lo siguiente:

- Las propuestas de investigación aprobadas a lo largo de la existencia de la COMI se enmarcan en alguna de las líneas de investigación de la ECSH. En otras palabras, mantienen una relación estrecha con la investigación en la sociedad, la cultura y el desarrollo.

- Los pre-diseños de investigación se han ajustado a ofrecer el mejor tratamiento al objeto de estudio con el objetivo de obtener nueva información que realmente responda la pregunta de investigación formulada desde el inicio del proceso. Se ha respetado el posicionamiento teórico y epistemológico desde donde la persona investigadora parte en la búsqueda del conocimiento.

- Los resultados obtenidos de las investigaciones han contado con la oportunidad de hacer la devolución respectiva a los entes que fueron investigados. Se ha salvaguardado la identidad e integridad de los sujetos participantes en las investigaciones.

- Como corolario, se ha de manifestar que dentro de las posibilidades actuales de investigación en la vida de la Escuela de Ciencias Sociales y Humanidades, se siguen planteando, y se aprobarán por la COMI, aquellas propuestas de investigación que promocionen y fomenten la investigación científica sobre la sociedad, la cultura y el desarrollo.

\section{Los resultados}

Una vez llevadas a cabo las acciones descritas, a la fecha, y según el orden en que fueron expuestas en el acápite anterior, se han obtenido los siguientes resultados:

- Se ha logrado reinsertar en el proceso de elaboración y defensa de TFG a más de cuarenta egresados, de diferentes cohortes.
Inicialmente, a través del período ventana, el cual se concedió para atender a las personas estudiantes de las cohortes anteriores a la novena, incluso, mitad de la octava, que no habían sido formadas con las destrezas y herramientas necesarias para enfrentar los nuevos protocolos de investigación que se estaban implementando. A ello se debe añadir que, en muchos casos, esta reincorporación supuso un esfuerzo adicional para las personas egresadas, ya que habían perdido mucha o toda la conexión que tenían con los ritmos, contenidos y hábitos propios de la educación a distancia, como producto del abandono que habían dado a su proceso de elaboración y defensa de TFG.

- Los talleres de pre-Investigación se han convertido en el espacio de pausa estratégica, análisis, intercambio y debate académico necesario para que las personas estudiantes lleven a cabo una escogencia adecuada del tema que desarrollarían para su TFG. Han sido el espacio de ensayo donde se construye, en un ir y venir entre la teoría, la práctica investigativa y la disciplina profesional, una propuesta de investigación sólida que se desarrolla a lo largo de los cursos EIJL1 y EIJL2. Se da también un avance en el proceso de elaboración del TFG, pues al iniciar la primera materia con la propuesta de investigación avanzada, el tiempo se aprovecha en lo que es el propósito del curso, cual es el avance significativo y perfeccionamiento de esa propuesta para, en EIJL2, finalizarla. De esta manera, la persona estudiante finaliza el plan de estudios con el informe borrador final de su TFG concluido, o prácticamente concluido, de forma que solamente le resta afinar los detalles para la defensa en el cuatrimestre que sigue.

- El Comité Asesor de la Persona Estudiante lo conforman una dirección de su TFG y una lectoría. Cuanto más temprano esté asignado y sea efectivamente acompañado el proceso, más fácilmente y mayor espacio para debate y mejoras se dará. De esta manera, 
al asignarse la dirección del TFG, la persona estudiante ya llega donde su guía con un planteamiento de investigación avanzado en su formulación, lo que le da una base lo suficientemente sólida y común para continuar el desarrollo. El profesor encargado de los cursos EIJL1 Y EIJL2, así como la Dirección de la MDTSS, tienen también una participación activa durante todo el proceso.

- El protocolo de elaboración y defensa de TFG logró llevarse a rango de normativa universitaria, actualmente está vigente bajo la denominación "Orientaciones para el desarrollo y presentación de los Trabajos Finales de Graduación en los Programas de Maestrías Profesionales" ${ }^{1}$. Esto permite no sólo dar certeza jurídica a las personas estudiantes y el comité asesor de lo que se espera, sino también dotar a los trabajos de estándares mínimos de forma y calidad, lo que contribuye a su aprehensión y divulgación.

- El tener un período límite para presentar y defender el TFG no sólo da rigurosidad al proceso, sino que obliga al postulante a ajustarse a los estándares de calidad y de resultados que se esperan de un graduado de posgrado. El límite temporal también sirve como una especie de control para evitar el aumento de los egresados y de estímulo sobre el número de personas graduadas.

- Se autorizó la apertura de los cursos EIJL1 Y EIJL2 fuera de su período regular, de forma tal que las personas estudiantes rezagadas de su plan de estudios por la desaprobación de ambos cursos y las personas que egresadas puedan reincorporarse al proceso de elaboración y defensa del TFG con los protocolos de investigación vigentes actualmente. Esto abre las posibilidades para lograr mayores porcentajes de investigación y graduación, además minimiza el porcentaje residual de personas sin concluir sus procesos académicos.

1. Consejo Universitario, Sesión 2268-2013, Art.II, inciso 1.a) 18 de julio.
- Además de formar parte del acervo bibliográfico institucional, pues los informes de los TFG se guardan en el Centro de Información, Documentación y Recursos Bibliográficos de la UNED, ya se han logrado articular otras formas de divulgación de las investigaciones de la MDTSS. Estas se han publicado en artículos científicos en revistas disciplinares y entrevistas en radio sobre los temas que las personas graduadas investigan. De la misma manera, hay productos más disciplinares, como Proyectos de Ley.

- El porcentaje de estudiantes que habiendo concluido el plan de estudios se gradúa aumentó en 5 puntos porcentuales entre 2011 y 2012 , pasando de $62,4 \%$ a $67,5 \%$. Este aumento no se debió a una mejora en el rendimiento académico del estudiantado, sino a la conclusión del TFG de estudiantes que se encontraban atrasados en este proceso.

(...) es importante subrayar por tanto que este incremento en el porcentaje de graduación tiene relación con las acciones ejecutadas desde la Coordinación de la MDTSS para que las personas rezagadas concluyeran su TFG.

Los porcentajes de graduación tienden a ser más altos y en una menor cantidad de tiempo en el caso de las mujeres. Del total de las que han ingresado, un 44,9\% se ha graduado, mientras que en el caso de los hombres, ese porcentaje resulta de apenas el $37,6 \%$ del total de los matriculados. Aunado a esto, un 62,26\% del total de mujeres que se han graduado invirtió menos de 25 meses entre ingresar a la Maestría y defender su TFG; en el caso de los hombres este porcentaje desciende a $37,5 \%$.

(...) En resumen, introduciendo la variable de sexo, se puede concluir que el grupo de graduados ha tendido a estar conformado mayoritariamente por mujeres, que se gradúan en menos de 25 meses y que tienen una opinión positiva respecto de la MDTSS. (Vargas, 2012: 46)

\section{Las mejoras a realizar}

Como en todo proceso de mejora continua existen retos que cumplir. El primero de ellos es 
dar el último paso en el protocolo de investigación vigente, el cual se resume en capacitar a los miembros de los comités asesores de las personas estudiantes, en los detalles y procesos innovadores que se han ido poniendo en práctica. Parte de la dinamización del proceso incluye la apertura de los cursos EIJL1 Y EIJL2 en forma extraordinaria y no sólo cuando el desarrollo curricular de la cohorte activa lo indique, en forma tal que se amplíen las posibilidades de las personas egresadas y rezagadas en presentar sus TFG y acortar sus tiempos de graduación.

Otra de las debilidades que se han detectado es la ausencia o insuficiencia de recursos para la investigación disciplinar. Esto, unido a la necesidad de dar a conocer lo que se está haciendo en la MDTSS en esta materia, así como la innovación en los procesos pedagógicos, a través de una mayor interacción entre las personas estudiantes y profesoras han creado las condiciones propicias para incorporar a la MDTSS los llamados "Recursos Educativos Abiertos". "Los recursos educativos de libre acceso son materiales de enseñanza, aprendizaje o investigación que se encuentran en el dominio público o que han sido publicados con una licencia de propiedad intelectual que permite su utilización, adaptación y distribución gratuitas" (UNESCO, 2012)

Actualmente, se están trabajando varios procesos de elaboración y divulgación de la investigación generada en la MDTSS, a través de este tipo de recursos. Estos dan un mayor énfasis a la incorporación de la innovación educativa en ese posgrado.

\section{Conclusión}

A través de sus quince años de existencia, la MDTSS ha pasado por varias etapas. Actualmente su trayectoria académica, la de sus estudiantes, graduados y docentes, permite una proyección y un impacto más importante en su quehacer. El resultado final del proceso académico es la investigación, materializada en los TFG. Cuanto más cantidad, mejor calidad y más abiertos sean, más escalable será su valor y su devolución a la sociedad costarricense, la cual es la que financia la universidad pública a la que pertenece la persona estudiante. La misión y la visión de la UNED, así como la de la MDTSS misma obligan, cada vez más, a llevar adelante los procesos de mejora continua que aquí se exponen.

\section{Referencias bibliográficas}

Abello, Raimundo. (2009, junio) La investigación en Ciencias Sociales: sugerencias prácticas sobre el proceso. vol. 17, no. 1 disponible en: http://rcientificas.uninorte.edu.co/ index.php/investigacion/issue/view/51

CAntero, Fabiola (2013) Construyendo la calidad: resultados preliminares en el caso de la Maestría en Derecho del Trabajo y Seguridad Social de la Universidad Estatal a Distancia (UNED) En Revista Calidad en la Educación Superior (4) 1. Dirección electrónica: http://web.uned. ac.cr/revistas/index.php/caes/article/view/140/168 Revisado la última vez: 28 febrero, 2015.

CONARE (1982) Convenio de Coordinación de la Educación Superior Universitaria Estatal en Costa Rica. Dirección electrónica: http://www.cu.ucr.ac.cr/normativ/convenio_conare.pdf Revisado la última vez: 15 enero, 2015.

RAMírez, Jorge (2011). Como diseñar una investigación académica. San José: Montes de María Editores.

UNED (2014) Reglamento General Estudiantil. Dirección electrónica :http://www.uned.ac.cr/academica/images/ cidreb/reglamento/estudiantil/general_estudiantil.pdf

Revisado por última vez: 28 febrero, 2015

UNED (2014) Reglamento del Sistema de Estudios de Posgrado. Dirección electrónica: http://www.uned.ac.cr/ academica/images/cidreb/reglamento/estudiantil/sistema_estudios_posgrado.pdf. Sep. Revisado por última vez: 2 marzo, 2015

Umaña, R. (2011) Análisis de las dificultades que presentan los estudiantes que abandonan, interrumpen, se egresan y se gradúan de la Maestría en Derecho de Trabajo y de la Seguridad Social. San José: CR-EUNED

Umaña, R., Vargas, K. (2012) Análisis de las dificultades que presentan los estudiantes que abandonan, interrumpen, se egresan y se gradúan de la Maestría en Derecho de Trabajo y de la Seguridad Social. San José: CR-EUNED

UNESCO (2014) Recursos Educativos Abiertos. Dirección electrónica: http://www.unesco.org/new/es/communication-and-information/access-to-knowledge/open-educational-resources/ Revisada por última vez: 15 enero. 2015 . 